\title{
Modeling the hydrodynamics and the process of averaging a highly concentrated granular medium in a mixing hopper
}

\author{
Alexander Shvab, Anastasia Martsenko, and Maksim Martsenko ${ }^{a}$ \\ Tomsk State University, Applied Aeromechanics Department, 36, Lenin Avenue, 634050 Tomsk, \\ Russia
}

\begin{abstract}
The study of motion and mixing of a highly concentrated loose granular medium in a vertical mixing hopper in the inertial motion mode within the framework of the "theory of fast granular flows" are shown.
\end{abstract}

This paper presents the study of motion and mixing of a highly concentrated loose granular medium in a vertical mixing hopper in the inertial motion mode [1] within the framework of the "theory of fast granular flows". To describe the flow, a differential model based on continuum mechanics concepts and rheological properties of granular materials with an additional equation of chaotic energy transfer caused by pulsation of the granules [2] is used. Simulation of the mixing process is based on the transport equation of concentration of the primary and key components of the granular mixture. Energy of the stochastic motion of granules in the inertial flow regime is modeled by analogy with the theory of turbulence [3]. Energy dissipation of the stochastic motion of the granular medium and determination of the viscosity of the model in the equations of momentum and energy transport are written by means of the Kolmogorov-Prandtl formula. Granular motion in the pulsating mode is limited due to the high concentration of the granular medium that allows, in the first approximation, to consider the length scale of the chaotic pulsation of the granules as a constant value.

This paper focuses on the steady gravity flow of a highly concentrated granular medium in a vertical silo with horizontal plates inside for intensive mixing of the primary and key components of the mixture. A scheme of such a bunker is shown in Fig. 1. At the entrance to the channel an unmixed homogeneous (by its physical properties) granular medium is fed at a constant speed from the top. The process of averaging is due to convection and diffusion phenomena caused by the chaotic pulsation of the granules in the inertial flow mode when moving through the bunker.

To describe the hydrodynamics and the averaging process of a highly concentrated granular medium in the inertial flow regime, a set of equations consisting of those of momentum transport, the continuity equation, the equation of stochastic energy of pulsating granular motion and the transport equation of

\footnotetext{
${ }^{a}$ Corresponding author: martsenko@sibmail.com

This is an Open Access article distributed under the terms of the Creative Commons Attribution License 4.0, which permits unrestricted use, distribution, and reproduction in any medium, provided the original work is properly cited.
} 


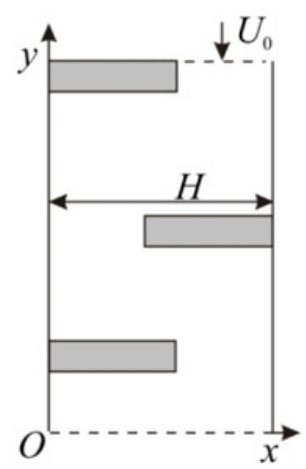

Figure 1. Vertical mixing hopper.

concentration for the key component of the mixture:

$$
\begin{aligned}
& \frac{\partial\left(u_{x}^{2}\right)}{\partial x}+\frac{\partial\left(u_{x} u_{y}\right)}{\partial y}=-\frac{\partial p}{\partial x}+\frac{\partial}{\partial x}\left(\frac{(1+b)}{\operatorname{Re}} \frac{\partial u_{x}}{\partial x}\right)+\frac{\partial}{\partial y}\left(\frac{(1+b)}{\operatorname{Re}} \frac{\partial u_{x}}{\partial y}\right)+ \\
&+\frac{1}{\operatorname{Re}}\left(\frac{\partial b}{\partial x} \frac{\partial u_{x}}{\partial x}+\frac{\partial b}{\partial y} \frac{\partial u_{y}}{\partial x}\right) ; \\
& \frac{\partial\left(u_{x} u_{y}\right)}{\partial x}+\frac{\partial\left(u_{y}^{2}\right)}{\partial y}=-\frac{\partial p}{\partial y}+\frac{\partial}{\partial x}\left(\frac{(1+b)}{\operatorname{Re}} \frac{\partial u_{y}}{\partial x}\right)+\frac{\partial}{\partial y}\left(\frac{(1+b)}{\operatorname{Re}} \frac{\partial u_{y}}{\partial y}\right)+ \\
&+\frac{1}{\operatorname{Re}}\left(\frac{\partial b}{\partial x} \frac{\partial u_{x}}{\partial y}+\frac{\partial b}{\partial y} \frac{\partial u_{y}}{\partial y}\right) ; \\
& \frac{\partial\left(u_{x} e\right)}{\partial x}+\frac{\partial\left(u_{y} e\right)}{\partial y}=\left.\frac{1}{\operatorname{Re}}\left\{\frac{\partial u_{x}}{\partial x}+\frac{\partial u_{y}}{\partial y}=0 ;(1+b) \frac{\partial e}{\partial x}\right]+\frac{\partial}{\partial y}\left[(1+b) \frac{\partial e}{\partial y}\right]\right\}+ \\
&+\frac{b}{\operatorname{Re}}\left[2\left(\frac{\partial u_{x}}{\partial x}\right)^{2}+2\left(\frac{\partial u_{y}}{\partial y}\right)^{2}+\left(\frac{\partial u_{x}}{\partial y}+\frac{\partial u_{y}}{\partial x}\right)^{2}\right]-c_{2} \frac{e^{3 / 2}}{l} \\
& u_{x} \frac{\partial C}{\partial x}+u_{y} \frac{\partial C}{\partial y}=\frac{1}{\operatorname{ReSc}}\left(\frac{\partial^{2} C}{\partial x^{2}}+\frac{\partial^{2} C}{\partial y^{2}}\right)
\end{aligned}
$$

where

$$
b=\frac{c_{1}}{\operatorname{Re}} l \sqrt{e} ; \quad \operatorname{Re}=\frac{U_{0} H}{B_{0}} ; \quad \mathrm{Sc}=\frac{B_{0}}{D} ; \quad e=\frac{E}{U_{0}^{2}} ; \quad b=\frac{B}{B_{0}} ; \quad u_{x}=\frac{U_{x}}{U_{0}} ; \quad u_{y}=\frac{U_{y}}{U_{0}} ; \quad l=\frac{L}{H} .
$$

Here $L$ - the length scale of the chaotic pulsation of the granules which can be taken constant because of the high concentration of the granular medium; Re H Sc - the Reynolds and Schmidt numbers, respectively; $c_{1}$ and $c_{2}$ - constants of the model; $B$ - the kinematic viscosity coefficient of the model, taking into account the additional momentum transfer due to pulsating motion of the granular medium and $B_{0}$ - the constant of viscosity for loose granular materials which can generally depend upon the stress in the granular medium. In this paper $B_{0}$ is taken to be constant. 

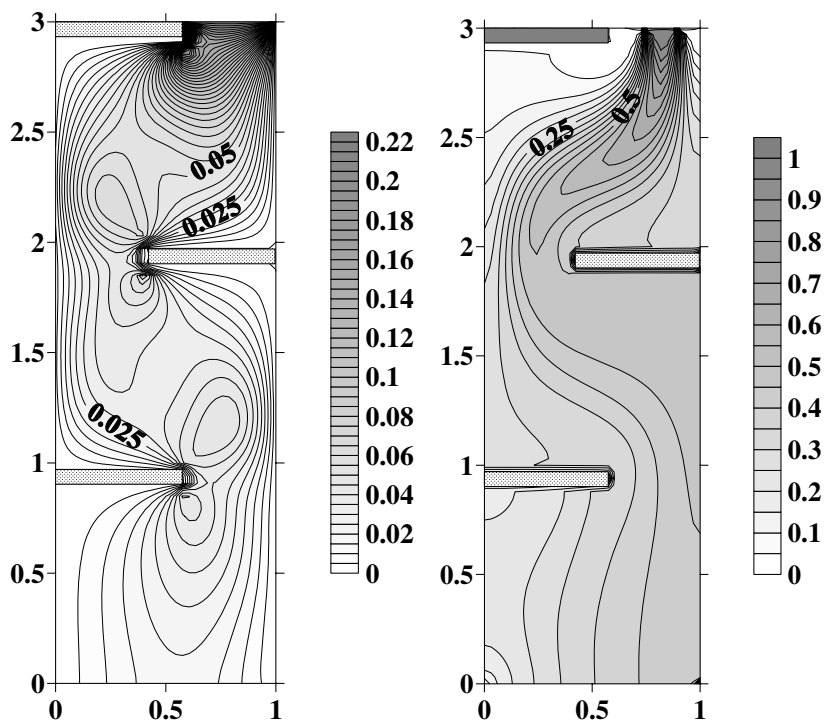

Figure 2. Kinetic energy distribution of and distribution of concentration.

To obtain a unique solution equations system (1-5), the following boundary conditions are applied. In the inlet section for the dimensionless values of the vertical and horizontal components of the velocity vector it is supposed that $u_{y}=-1, \partial u_{x} / \partial y=0$. On the channel walls the normal velocity component is zero, and for the tangential component of the velocity the slip condition applies. It is assumed that the fluid velocity at the wall is determined not only by the magnitude of the stress tensor, but also by the dry Coulomb friction of the wall-particle pair. Therefore, to determine the slip conditions an independent empirical parameter is introduced whose value is determined by comparing the numerical and experimental data. In dimensionless form, the condition of partial slip on the wall for the granular medium can be written as

$$
\frac{\partial u_{s}}{\partial n}=\beta u_{n}
$$

where $n$ - the normal to the wall; $u_{s}$ - the tangential component of the velocity vector; $\beta=\beta^{0} / H$ - the dimensionless independent empirical coefficient $\left(\beta^{0}-\right.$ the scaled slip ratio) whose value is in the range $0 \leq \beta \leq \infty$, and the value of $\beta=0$ corresponds to the condition of full slip medium on the wall and $\beta \rightarrow \infty$ corresponds to the slip condition. For better agreement between the theoretical calculations and experimental data on the front of the plates in the direction of the flow the adhesion conditions are used for the velocity components, and on the other borders - the condition of partial slip (6). Kinetic energy $e$ at the wall is zero, and its inlet value is taken as a constant. At the upstream boundary in the central zone the key component concentration of the mixture is defined by the constant value, $C=1$. At the periphery of the same cross section concentration of the main component is given, which is equal to zero. On the walls there is no flow concentration $(\partial C / \partial n=0)$. The outlet boundary conditions apply $\partial / \partial y=0$ for all functions in question.

Figure 2 on the left shows the distribution of isolines of the averaged kinetic energy of the stochastic pulsating motion through the bunker assuming: $\operatorname{Re}=10 ; c_{1} \cdot l=1.5 ; c_{2} / l=10.5 ; \beta=0$. The field of concentration isolines for the key component of the granular mixture is shown in Fig. 2 on the right. From this graph it can be clearly seen how the process of averaging the mixture occurs, taking into account that mixing is completed at the exit of the hopper. 


\section{EPJ Web of Conferences}

This work was financially supported by the RFBR grant 13-08-00372-a.

\section{References}

[1] Mechanics of Granulated Media. The Theory of Fast Granular Flows, Collected papers, Mechanics (Russian translation, Mir, Moscow, Issue 36, 1985)

[2] A.A. Martsenko, M.S. Martsenko, N. S. Shvab, Izv. Vyssh. Uchebn. Zaved., Fizika, 55, 7, P. 115$118,(2012)$

[3] G. Ahmadi, M. Shahinpoor, Powder Technol., 35, 2, P. 241-248. (1983) 